\title{
Crack arrest and propagation in impact loaded shock resistant PMMA: mesh-free numerical simulation
}

\author{
Kean-Sheng $\operatorname{Tan}^{1}$, Patrice Longère ${ }^{2 *}$, and Norazrina Mat-Jali ${ }^{1}$ \\ ${ }^{1}$ Faculty of Engineering, National Defence University of Malaysia, 57000 Kuala Lumpur, Malaysia \\ ${ }^{2}$ Institut Clément Ader, Université de Toulouse, ISAE-SUPAERO, MINES ALBI, UPS, INSA, \\ CNRS, 31400 Toulouse, France
}

\begin{abstract}
The use of shock resistant RT-PMMA in engineering structures potentially subject to accidental overloading requires an evaluation of its crack arrest capability under impact loading. Based on experimental results obtained from a series of Kalthoff and Winkler (KW)-type impact tests, the present study aims at numerically reproducing the conditions for brittlelike crack initiation and propagation in impact-loaded RT-PMMA. For that purpose, three-dimensional SPH numerical simulations were conducted and the performance of various failure criteria was evaluated. The numerical model together with a combination of stress- and straindependent failure criteria were shown to fairly reproduce the experimental results in terms of finite crack advance and orientation.
\end{abstract}

\section{Introduction}

A way of improving the poor impact resistance of PMMA consists in embedding rubber nano-particles inside a PMMA matrix to form the so-called rubber toughened PMMA or RT-PMMA, see [1]. The use of shock resistant RT-PMMA in engineering structures, as f.ex. protection window, requires an evaluation of its crack arrest capability under impact loading. For that purpose, a series of Kalthoff and Winkler (KW)-type impact tests [2-3] has been carried on several grades of RT-PMMA evidencing the role of the rubber nanoparticles concentration, see [4,5]: the higher the nano-particles concentration the higher the resistance to crack propagation.

Based on these tests, the present study aims at numerically reproducing the conditions for brittle-like crack initiation and propagation in impact-loaded pre-cracked RT-PMMA. A particular attention is paid to reproduce the crack arrest, in terms of finite crack advance and orientation, which is far from being trivial and accordingly rarely addressed in literature.

As an alternative to standard finite element method (FEM)-based numerical simulations, see [6-8], known to involve unavoidable mesh-dependent crack path, the smooth particle hydrodynamics (SPH)-based method was preferred here, see [9]. Various stress- and straincontrolled failure criteria were evaluated regarding their performances to reproduce the

* Corresponding author: patrice.longere@isae.fr 
dependence of the finite crack advance and orientation on impact velocity as observed experimentally. A parametric study was then achieved to calibrate the adopted failure criterion.

Some experimental results are recalled in Section 2 and the numerical procedure is detailed in Section 3. The results obtained with the adopted failure criterion are shown and commented in Section 4. Concluding remarks are finally given in Section 5.

\section{Experimental results}

$\mathrm{KW}$-impact tests consist in impacting the edge of a doubly-notched plate made of the material of interest with a cylindrical projectile. Depending on the plate material and impact velocity magnitude, cracks may initiate at the notches tips with a kink angle of $+/-70^{\circ}$ wrt to the notch plane (brittle fracture), see e.g. [2], or in a self-similar way (ductile fracture under adiabatic shear banding), see e.g. [3].

A series of KW-type impact tests were carried out on a class of RT-PMMA at various impact velocities at ICA Lab. using the STIMPACT facility equipped with three singlestage gas guns. The $40 \mathrm{~mm}$-diameter gas gun was used. The KW-type specimen and the sub-calibrated projectile with its guide (sabot) are shown in Fig.1. The plate/projectile interaction was video-recorded at $100 \mathrm{kfps}$ using a high-speed camera, see an example of picture in Fig.2a.

The tests showed that the cracks initiated from the notches tips with an angle of $+/-70^{\circ}$ wrt to the notch plane, see Fig.2 (and Fig.3a), as expected for a semi-brittle material, and that the higher the impact velocity the larger the number of fragments. Moreover, within the impact velocity range $[20 \mathrm{~m} / \mathrm{s} ; 40 \mathrm{~m} / \mathrm{s}]$, cracks initiated from the notches tips are seen to arrest inside the specimen, with a crack advance depending on the impact velocity, see Fig.2.

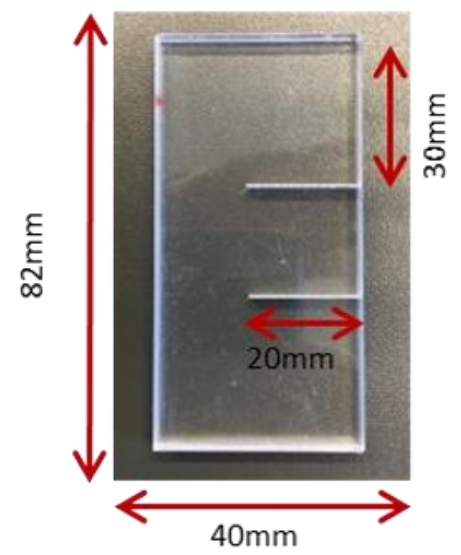

a) KW-type doubly-notched specimen

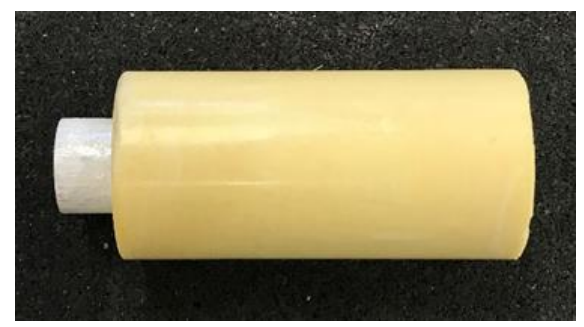

b) Sub-calibrated projectile and its sabot

Fig. 1. KW-type specimen and projectile used for the KW-type impact tests. After [5]

\section{Numerical procedure}

Numerical simulations were conducted using the industrial computation code LS-DYNA employing the three-dimensional SPH-based spatial discretization and the explicit time integration scheme. 


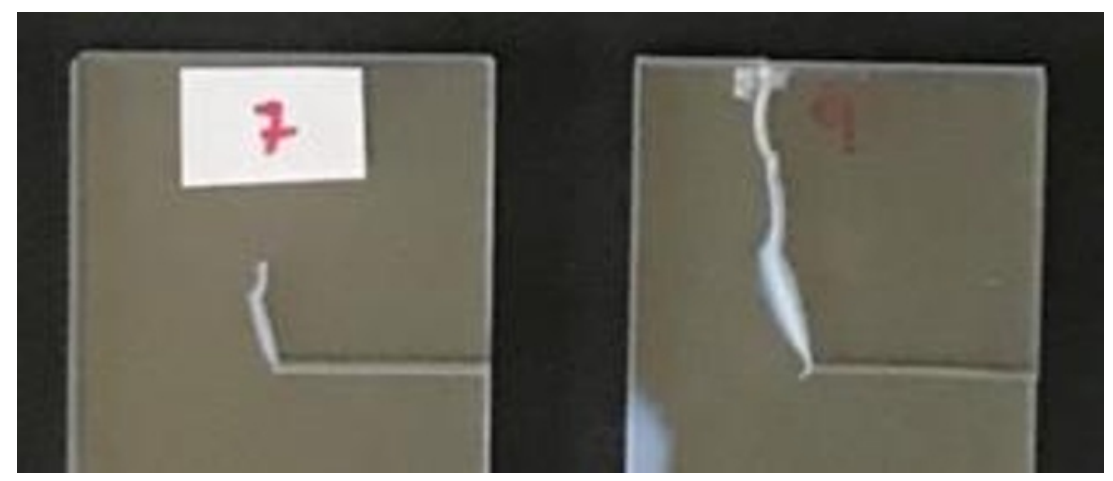

Fig. 2. Experimental results after impact velocity of $25 \mathrm{~m} / \mathrm{s}$ (left) and $30 \mathrm{~m} / \mathrm{s}$ (right). Evidence of crack arrest inside the plate. After [5]

In order to be as close to the experimental conditions as possible, all the parts interacting during the impact were modeled. Both RT-PMMA doubly-notched plate and steel sub-calibrated projectile were discretized using particles (SPH), and the polyethylene foam projectile guide (sabot) using finite elements, see Fig. $3 \mathrm{~b}$.

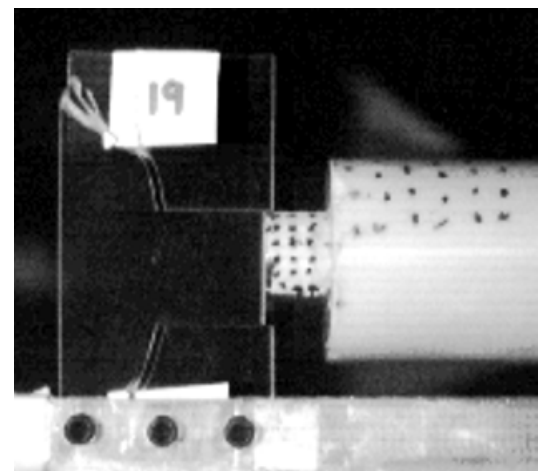

a) Experimental test. After [5]

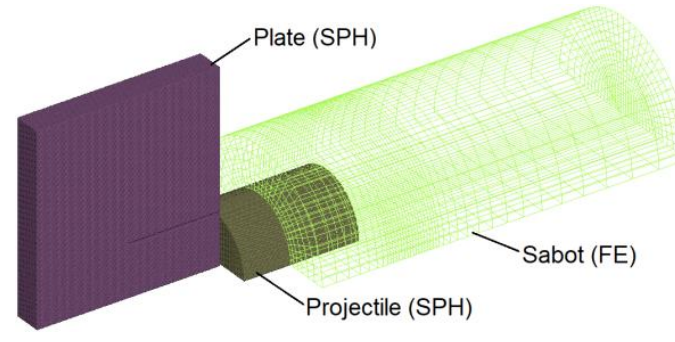

b) Numerical model

Fig. 3. Experimental configuration and numerical model.

The rate- and temperature-dependence of the RT-PMMA behavior was described using tabulated curves coming from [5], as were the steel and foam behaviors. Self-heating was accounted for by considering a Taylor-Quinney coefficient of 1 .

To palliate the pathological SPH issue related to tensile instability and account for small to moderate deformation, the regularised Lagrangian approximation was used. The penetration was minimised thanks to the combination of the SPH contact condition and time scale factor.

\section{Numerical results}

The performances of stress- and strain-dependent failure criteria regarding their ability to reproduce the overall crack pattern and arrest were investigated, by first considering each criterion individually, and by then combining them. 


\subsection{Stress- vs strain-dependent failure criterion}

Failure criteria for brittle materials are generally expressed in terms of critical maximum principal stress (CMPS), see [9]. For the RT-PMMA under consideration, the maximum principal stress corresponding to a crack initiation at an impact velocity near $20 \mathrm{~m} / \mathrm{s}$ is close to $103 \mathrm{MPa}$. This value has accordingly been taken as CMPS. Corresponding numerical simulations at the impact velocities $25 \mathrm{~m} / \mathrm{s}$ and $30 \mathrm{~m} / \mathrm{s}$ are shown in Fig. 4 . According to the latter, the cracks initiate from the notches tips with the expected angle $+/-70^{\circ}$ but cross the whole plate. In other words, the crack orientation is well reproduced whereas the crack arrest is not.

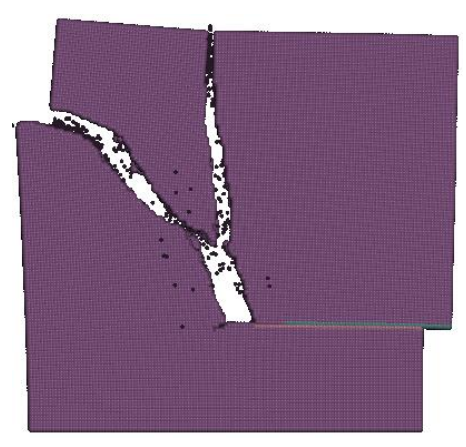

a) $25 \mathrm{~m} / \mathrm{s}$

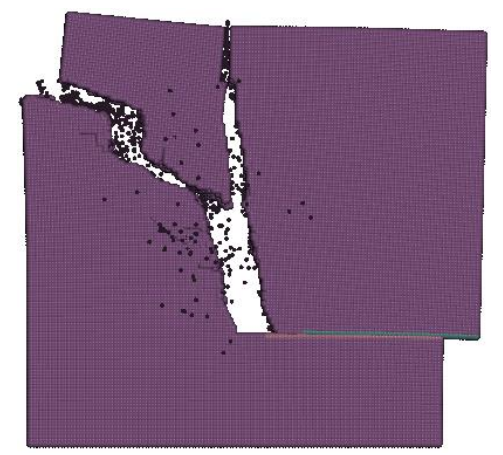

b) $30 \mathrm{~m} / \mathrm{s}$

Fig. 4. Numerical simulations. Maximum principal tress-dependent failure criterion. CMPS=103MPa. To be compared with Fig.2.

In parallel, failure criteria for ductile materials are generally expressed in terms of critical equivalent plastic strain (CEPS), see [6]. For the RT-PMMA under consideration, the equivalent plastic strain corresponding to a crack initiation at an impact velocity near $20 \mathrm{~m} / \mathrm{s}$ is close to $2.9 \times 10^{-2}$. This value has accordingly been taken as CEPS. Corresponding numerical simulations at the impact velocities $25 \mathrm{~m} / \mathrm{s}$ and $30 \mathrm{~m} / \mathrm{s}$ are shown in Fig.5. According to the latter, the cracks initiate from the notches tips with the expected angle $+/$ $70^{\circ}$ then arrest inside the plate, while unexpected cracks propagate downwards in the middle of the plate (see Fig.5b). In other words, the crack orientation and arrest are well reproduced whereas other cracks form.

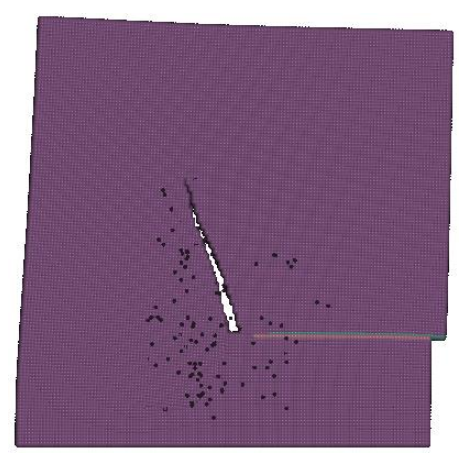

a) $25 \mathrm{~m} / \mathrm{s}$

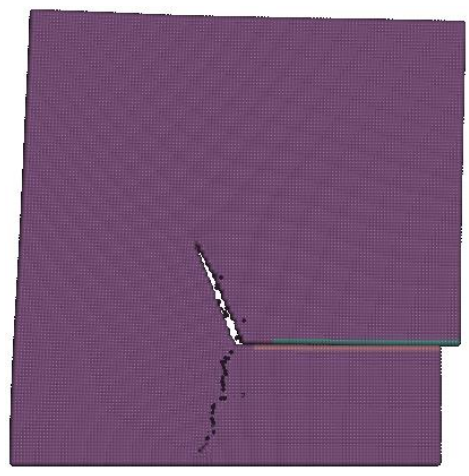

b) $30 \mathrm{~m} / \mathrm{s}$ (middle of the plate)

Fig. 5. Numerical simulations. Equivalent plastic strain-dependent failure criterion. $C E P S=2.9 \times 10^{-2}$. To be compared with Fig. 2. 
As above shown, the use of stress- and strain-dependent failure criteria individually fails to reproduce the real crack conditions and pattern in the RT-PMMA of interest under impact loading.

\subsection{A combination of stress- and strain-dependent failure criteria}

The objective is here to evaluate a failure criterion according to which failure occurs if and only both CMPS and CEPS are reached. Following a design of experiment, a parametric study was conducted to find the best set of (CMPS, CEPS) pair leading to the values reported in Table 1.

Table 1. Best set of (CMPS,CEPS) pair for the RT-PMMA under consideration.

\begin{tabular}{|c|c|}
\hline CMPS (MPa) & 115 \\
\hline CEPS & $5 \times 10^{-2}$ \\
\hline
\end{tabular}

Three-dimensional numerical simulations conducted with the set of (CMPS, CEPS) pair in Table 1 are shown in Fig.6. When compared with Fig.2, one can conclude that the numerical finite crack advance and orientation and overall crack pattern match fairly the experimental ones.

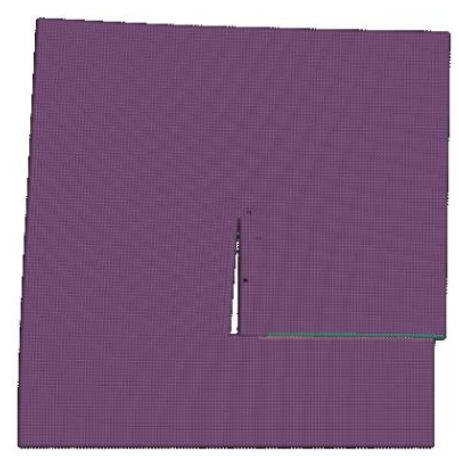

a) $25 \mathrm{~m} / \mathrm{s}$

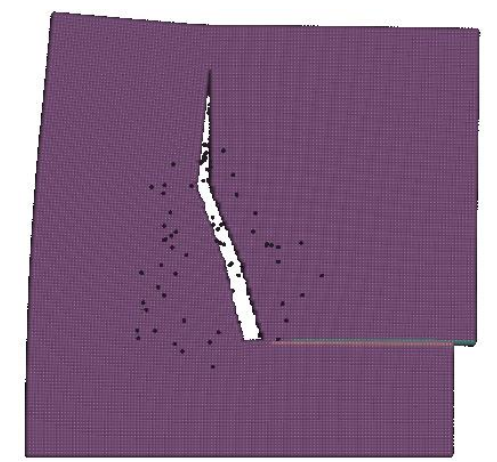

b) $30 \mathrm{~m} / \mathrm{s}$

Fig. 6. Numerical results after impact velocity of $25 \mathrm{~m} / \mathrm{s}$ (left) and $30 \mathrm{~m} / \mathrm{s}$ (right). To be compared with Fig.2.

\section{Concluding remarks}

In order to reproduce the experimental conditions for crack propagation and arrest in impact-loaded pre-cracked shock-resistant RT-PMMA, a three-dimensional SPH-based model was built, alleviating the well-known SPH tensile instability issue and accounting for the rate- and temperature-dependent nature of the material of interest. It was shown that the use of stress- and strain-dependent failure criteria individually fails to reproduce the real crack conditions and pattern in the semi-brittle RT-PMMA of interest. By contrast, the finite crack advance and orientation and the overall crack pattern can be fairly reproduced by combining the above mentioned criteria.

This numerical methodology can be applied to a wide range of semi-brittle polymers exhibiting crack arrest under impact loading. 


\section{References}

1. J. Wang, X. Zhang, L. Jiang, J. Qiao, Advances in toughened polymer materials by structured rubberparticles, Progress Polymer Sci., 98 (2019)

2. J.F. Kalthoff, S.Winkler, Failure mode transition of high rates of shear loading, in: C.Y. Chiem, H,-D. Kunze, L.W. Meyer (Eds), Proceedings of the International Conference on Impact Loading and Dynamic Behavior of Materials, Deutsche Gesellschaft fur Metallkunde, DGM-Verlag, Oberursel, Germany (1987)

3. E. Roux, P. Longère, O. Cherrier, T. Millot, D. Capdeville, and J. Petit, Analysis of ASB assisted failure in a high strength steel under high loading rate, Mater. Des., 75 (2015)

4. G. Manar, N. Mat Jali, P. Longère, Dynamic crack arrest capability of some metallic alloys and polymers, Proceedings of DYMAT 2018 Arcachon, EPJ Web of Conferences 183, (2018)

5. N. Mat Jali, N.M. and Longère, P., Experimental investigation of the dynamic fracture of a class of RT-PMMAs under impact loading, Int. J. Fracture, 225 (2020)

6. M. Zhou, AJ Rosakis, G. Ravichandran, Dynamically propagating shear bands in impact-loaded prenotched plates-II. numerical simulations, Journal of the Mechanics and Physics of Solids, 44 (1996)

7. RC. Batra, MVS. Ravinsankar. Three-dimensional numerical simulation of the Kalthoff experiment, Int. J. Fracture, 105 (2000)

8. K. Ravi-Chandar, J. Lu, B. Yang, Z. Zhu . Failure mode transitions in polymers under high strain rate loading, Int. J. Fract, 101 (2000)

9. S. Raymond, V. Lemiale, R. Ibrahim, R. Lau. A mesh free study of the KalthoffWinkler experiment in $3 D$ at room and low temperatures under dynamic loading using viscoplastic modelling, Engineering Analysis with Boundary Elements, 42 (2014) 